\title{
Cryo Field Emission Scanning Electron Microscopy Examination of the Ultrastructures of Agarose Based Chromatography Beads
}

\author{
David A. Bell*, Christopher Santeufemio* \\ *Electron Microscopy Lab, Millipore Corporation, 80 Ashby Rd. Bedford, MA 01730
}

Imaging agarose based chromatography beads using conventional high vacuum electron microscopy is problematic at best. This is because the delicate nature of their structure requires them to remain in a wet state or they will collapse. Cryo preparation of the samples allows the structures to maintain their integrity while making them visible for secondary electron imaging. This presentation will detail the different attempts made to examine these agarose structures and the final process that provides superior results. Some of the fascinating structures observed will also be highlighted.

Initial attempts at preparing the beads for microscopy were made by trying a solvent drying technique, similar to the routines used for the preparation of biological samples. There is some structure evident in these images (Figure 1), however it is somewhat flattened with very little porosity. The cryomicroscopy technique later demonstrated this structure to be artifacts of the preparation, likely caused by the collapse of the bead.

The cryomicroscopy approach is a labor-intensive process, yet the results achieved are remarkable. A sample of beads is first suspended in water. A small drop of the suspension is placed in a gold specimen carrier then plunge-frozen in liquid ethane. Using the Gatan CT-3500 cryostage (Figure 2), the frozen sample is maintained at liquid nitrogen temperatures $\left(\approx-180^{\circ} \mathrm{C}\right)$ and placed in a turbo pumped chromium coater. By warming the sample to $\approx-105^{\circ} \mathrm{C}$ while under a vacuum in the range of $10^{-7} \mathrm{Torr}$, the excess ice is sublimed off at a controlled rate, maintaining the structure of the beads. The sample is then coated with chromium, placed into the upper stage of a Topcon DS-150F field emission SEM (Figure 3) and imaged. This technique makes it possible to image the surface ultrastructure of the agarose beads (Figure 4), therefore the structural differences between various agarose bead formulations becomes apparent. Alternatively, before transferring the sample to the stage, the surface of the frozen drop can be scratched, causing several beads to fracture. This enables the visualization of the internal or cross-sectional structure of the bead.

This cryo preparation technique has proven its utility in an application where the samples have a need to remain hydrated and are delicate. It will prove useful in imaging a wide range of similar samples in the future. 


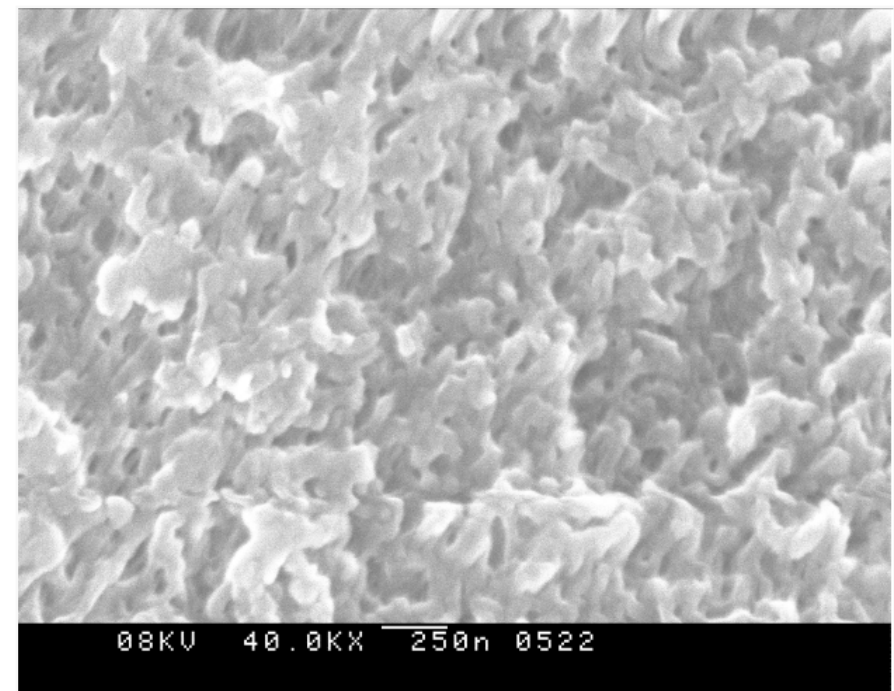

Figure 1: Surface of agarose bead prepared with solvent dehydration process showing some structure.

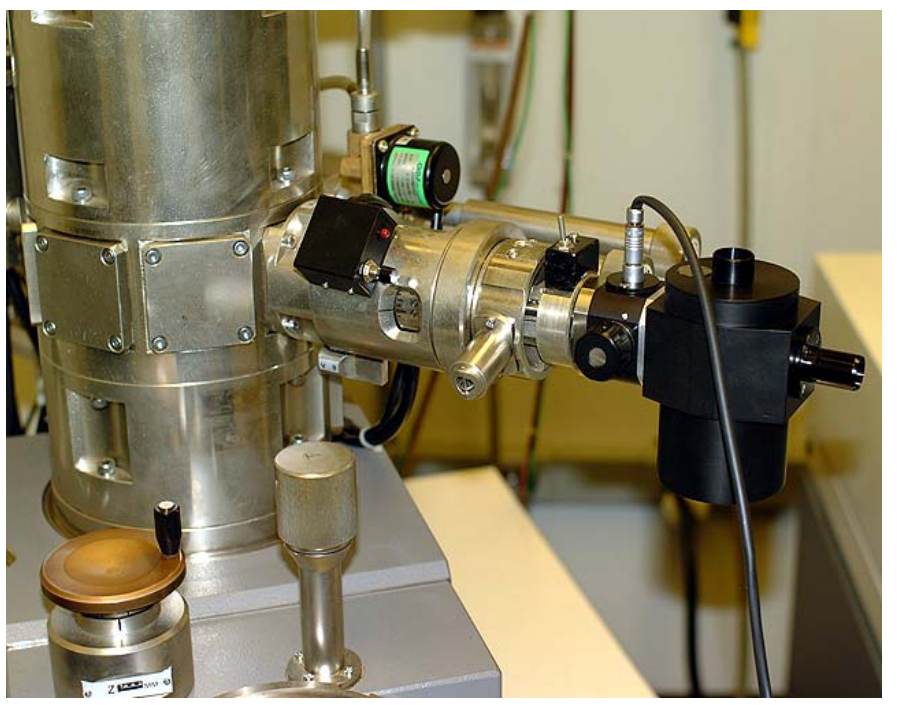

Figure 3: Cryostage mounted in the upper stage goniometer of the Topcon DS-150F FESEM.

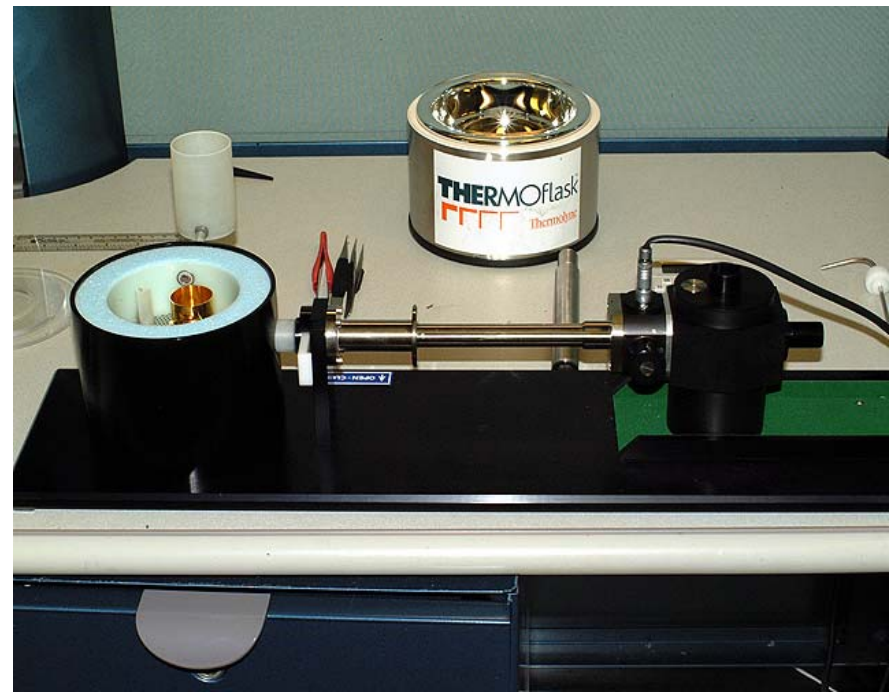

Figure 2: Gatan CT-3500 cryostage mounted on the sample transfer station.

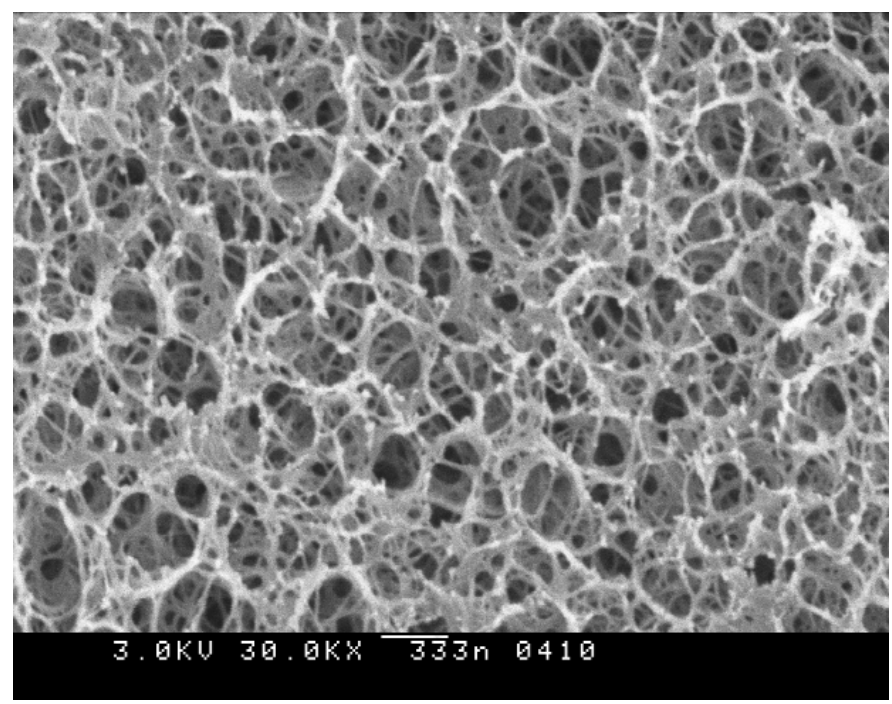

Figure 4: Surface ultrastructure of agarose bead revealed by cryomicroscopy. 